HUANG YAN, Ph.D. candidate ${ }^{1}$

E-mail: tjyh@tongji.edu.cn

XIAONING ZHANG, Ph.D. ${ }^{1}$

(Corresponding Author)

E-mail: cexzhang@tongji.edu.cn

${ }^{1}$ School of Economics and Management

Tongji University

Shanghai 200092, China
Transport Logistics

Original Scientific Paper

Submitted: 17 July 2020

Accepted: 11 Jan. 2021

DOI: $10.7307 /$ ptt.v33i4.3666

\title{
MEGAREGIONAL PASSENGER TRANSPORTATION HUB LOCATION PROBLEM CONSIDERING CONGESTION EFFECTS
}

\begin{abstract}
The need to make effective plans for locating transportation hubs is of increasing importance in the megaregional area, as recent research suggests that the growing intercity travel demand affects the efficiency of a megaregional transportation system. This paper investigates a hierarchical facility location problem in a megaregional passenger transportation network. The aim of the study is to determine the locations of hub facilities at different hierarchical levels and distribute the demands to these facilities with minimum total cost, including investment, transportation, and congestion costs. The problem is formulated as a mixed-integer nonlinear programming model considering the service availability structure and hub congestion effects. A case study is designed to demonstrate the effectiveness of the proposed model in the Wuhan metropolitan area. The results show that the congestion effects can be addressed by reallocating the demand to balance the hub utilisation or constructing new hubs to increase the network capacity. The methods of appropriately locating hubs and distributing traffic flows are proposed to optimise the megaregional passenger transportation networks, which has important implications for decision makers.
\end{abstract}

\section{KEYWORDS}

hierarchical hub facility location; megaregional passenger transportation; optimisation; service availability; hub congestion.

\section{INTRODUCTION}

From last century, rapid urbanisation processes have facilitated the formation of megaregions. Cities within a megaregion connected via transportation infrastructure have a high degree of economic and social integration and thus promote regional cooperation, which accelerates the flow of people between cities and regions [1]. Unlike that of intracity trips, the spatial distribution of intercity trips can be classified into area-level, regional-level, and national-level trips [2]. The hierarchical features of intercity passengers require multiple modes of transportation (air, railway, and bus) because each mode has a specific service range. For example, air transportation is particularly essential for international travel because there is no alternative travel mode for the long international trips. Therefore, an efficient megaregional transportation system needs to provide a full range of travel services accessible to all passengers. However, the high demand for intercity travel brings substantial pressure to the transportation networks. In China, the government has released a series of policies to guide the planning of megaregional passenger transportation infrastructure in which transportation hubs are the key nodes of a megaregional passenger transportation network. On the one hand, transportation hubs are places to access intercity travel services, and their layout is the foundation of the transportation structure. On the other hand, to reduce the operating cost, megaregional planning should achieve infrastructure sharing between cities by optimising the overall arrangement of passenger hub facilities. Therefore, this paper focuses on the passenger hub facility location problem at the megaregional scale.

Facility location problems (FLPs) have been extensively studied for planning the location of public infrastructure, such as hospitals, schools, and parking lots etc. Hubs are special facilities that provide a switching, sorting, and connecting function. Contreras and Fernández [3] pointed out the main differences between FLPs and hub location problems (HLPs). In FLPs, the service is given at the facilities, and there are no interactions between facilities. 
However, hub facilities interact with each other in HLPs, because the service is the demand allocation from origins to destinations via at least one hub (e.g. railway stations and airports). In this paper, a megaregional hub is a critical node of the network, not just a station or an airport. It is typically located at the junction of multiple links and usually has high levels of capacity, accessibility, and service. Therefore, we assume that a megaregional passenger hub can meet any travel demand of passengers as long as their destination is within the service range of the hub. Note that we focus only on the planning of the megaregional passenger hub, not all nodes of the transportation network. For the reasons given above, the megaregional passenger hub facility location problem is regarded as an FLP.

A megaregional passenger transportation system is a hierarchical system that has multiple types of passenger hubs that provide different transportation modes to meet the travel demand at different levels. Thus, the problem addressed in this paper needs to determine the locations of hub facilities at different levels and allocate the demand to these facilities. Furthermore, the existing network must be considered in the planning of a megaregional passenger transportation network. The existing hub facilities can be closed if these facilities fail to satisfy the economic principles of the plan. Nevertheless, when the problem aims to minimise the total construction and transportation cost, the plan would concentrate demand at a relatively small number of hubs. Thus, the number of passengers served by some hubs is likely to reach the full capacities of these passenger hubs, resulting in congestion and delays at the hubs. In fact, there may be no congestion effects only if a hub is working at less than a certain ratio of the capacity. For example, transportation infrastructure operating above $80 \%$ of nominal capacity usually starts to encounter congestion effects in the form of queuing at the hub's access points [4]. Moreover, even a relatively small increase in demand at a congested hub can cause more serious congestion issues. Hence, the hub facilities should be investigated considering these congestion effects.

In this paper, we present a hierarchical facility location model for a megaregional passenger transportation network design with three decisions: the construction of new hubs, the closing of existing hubs, and passenger allocation decisions. This model takes into account hierarchical travel demand, multiple level hubs, and congestion effects at hubs.
The objective function is the minimisation of the total cost, including investment cost, transportation cost, and congestion cost. A computational study is designed to test the performance of the proposed model for the Wuhan metropolitan area (WHM) located in eastern Hubei Province, Central China. Considering the regional differences in social and economic factors, the infrastructure investment cost and the hierarchical travel demand vary spatially. Based on the results of the congestion effect on the system, we finally propose two strategies to improve the network that have important implications for decision makers.

The main contribution of this paper is twofold. First, we propose a new service availability structure according to the relationship between intercity travel demand and hub facility service. This is the first paper that studies a facility location problem based on this structure, which makes it close to the real world. Second, the paper accommodates the hub congestion effects within a hierarchical hub location model using the power-law function. The proposed strategy satisfies the decision-making process in the real plan and reduces the total cost of the system as well.

The remainder of this paper is organised as follows. Section 2 provides a literature review. Section 3 presents the problem description and mathematical formulation. Section 4 then provides analysis results to demonstrate the performance of the proposed model as well as the strategies. Finally, Section 5 concludes the paper and discusses the future directions of this study.

\section{LITERATURE REVIEW}

The subject dealt with in this paper relates to two streams of research, namely hierarchical facility location problems (HFLPs) and congestion effects on hub facilities. In this section, a review of the hierarchical features of HFLPs and on the congestion effects incorporated in HLPs is given below. The reader is referred to Farahani et al. [5] and Farahani et al. [6] for a more extensive review on HFLPs and HLPs.

\subsection{Service availability}

In hierarchical systems, it is necessary to specify the availability of service provided by each type of facility for users at each level. Perhaps the earliest study in this context dates back to the work of 
Narula [7], who classified hierarchical systems into two types according to the service availability levels. In a successively inclusive system (also called a nested system), a facility provides its own level of service and all lower levels of service. This system is typically applied in a healthcare system where a higher-level hospital would offer a broader range of medical services, while a lower-level hospital would offer only specific services [8]. In a successively exclusive system (also called a non-nested system), a facility provides only its own level of service. For example, a facility (e.g. kindergarten, primary school, secondary school, and university) in an education system provides different stages of education service. Furthermore, there are distance restrictions relating the user to the facilities at levels, which means that a user only selects a candidate facility within an acceptable distance [9]. However, considering passenger transportation systems, no transportation mode can cover all ranges of passenger services, and different transportation modes might provide the same range of service. Hence, the service availability of a megaregional passenger transportation network is neither non-nested nor nested.

\subsection{Congestion effects}

Another significant addition to the megaregional passenger transportation network design literature is congestion. Grove and O'Kelly [10] were the first to study the effect of flow consolidation on hub congestion. They consider that airport congestion happens when the number of flights exceeds capacity, and congestion is one component of the decision-making process in HLPs. One way of mitigating congestion is to add a capacity constraint on hub flow. However, Marianov and Serra [9] stated that the usual capacity constraints do not reflect the dynamic nature of congestion, and they developed a spatial queuing system to simulate the quality-of-service conditions. Elhedhli and $\mathrm{Hu}$ [11] formulated the congestion effect at a hub using a convex cost function, which is the first work where congestion is incorporated in the objective function. This cost function is widely used to estimate delay cost, which is assumed to be a power-law function of capacity usage [12]. Later, Elhedhli and $\mathrm{Wu}$ [13] formulated the congestion at hubs as the ratio of total flow to surplus capacity and modelled a huband-spoke system as a network of $\mathrm{M} / \mathrm{M} / 1$ queues. De Camargo et al. [14] presented a congestion cost formulation similar to that of Elhedhli and $\mathrm{Hu}$ [11], but addressing the multiple allocation hub location problem. De Camargo et al. [15] redesigned the power-law congestion function to consider congestion effects only when a given flow threshold (also called capacity level) is exceeded. For example, there may be no congestion cost if a hub is utilising less than $80 \%$ of its capacity, whereas significant congestion may occur if a hub is utilising over $90 \%$ of its capacity. This new congestion function is also used in de Camargo and Miranda [16], Alumur et al. [17], and Özgün-Kibiroglu et al. [18]. Following de Camargo et al. [15], in this paper, we also model the congestion cost at a hub based on its capacity level.

In summary, there lacks systematic planning of transportation hubs at the megaregion level. The existing structure of service availability on most studies fails to account for the relationship between the multiple demands and megaregional hub service. Also, there is scarce literature that investigates the hub congestion effects on the HFLPs. In this paper, we aim to bridge these gaps by building a new service availability structure and taking the congestion factors into account in the megaregional passenger transportation hub location problem.

\section{PROBLEM FORMULATION}

\subsection{Service availability}

Megaregional passenger transportation networks are fundamentally hierarchical systems in which each level of the network can provide different services to meet the travel demand at various geographical scales. Service availability is one of the fundamental elements that can significantly affect the assignment of demand. We know that each transportation mode has its own features to serve specific travel demand, and distance is one of the basic determinants of modal usage for passenger transportation. Therefore, we define the level of a passenger hub by its service range. Similarly, the level of travel demand is determined based on travel distance. It is worth noting that there is an overlapping service area between hubs at two adjacent levels, which means that the demand within this area can be satisfied by two transportation modes. Considering the service availability of the hierarchical system, we build a crossed structure to represent the passenger accessibility to a service at different levels of hierarchy. Figure 1 shows one example of three service availability structures in a hierarchical system with three levels. Hubs in the non-nested 


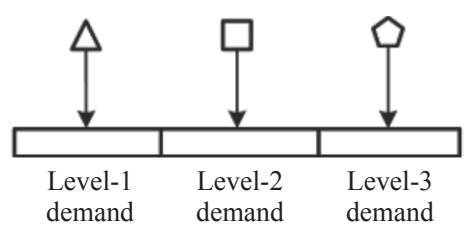

a) Non-nested structure

$\triangle$ Level-1 hubs $\square$ Level-2 hubs

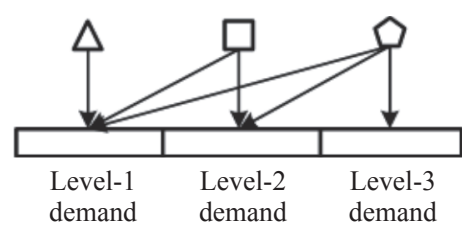

b) Nested structure

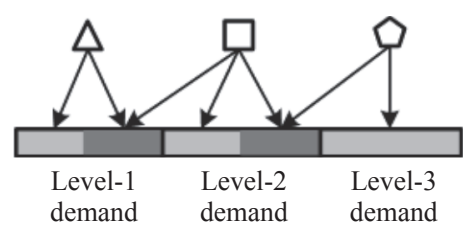

c) Crossed structure

Figure 1 - Examples of service availability in a hierarchical system with three levels

structure provide only their own level of service for the corresponding demand, whereas a higher-level hub in the nested structure provides all the services available at a lower-level hub plus an additional one. However, hubs in the crossed structure are neither non-nested nor nested. We have partitioned the travel demand into two types: $\{S, S H\}$. This categorisation ensures that $S$ demand must receive the same level of hub service, and $S H$ demand can be provided by hubs of the same level or exactly one level higher.

\subsection{Congestion effects}

Flow consolidation at passenger hubs achieves economies of scale but also causes congestion effects. Developing effective strategies to avoid congestion is appealing in improving the operational performance of a passenger hub system. Generally, the congestion cost increases more rapidly as more passengers are assigned to a hub. The closer the total demand approaches the hub capacity, the greater the congestion emerges. As mentioned before, the congestion cost functions available in the literature present a cost term that penalises excessive demand served by each hub, a power-law function [16] $P=\max \left\{0, e(G-\tau q)^{b}\right\}$ that considers congestion effects only after exceeding a given demand threshold $\tau q$ where $\tau$ (e.g., $70 \%, 80 \%, 90 \%$ ) represents the capacity level. $G$ represents the total demand served by a hub. $e$ and $b$ are positive constants with $b \geq 1$.

Figure 2 illustrates the power-law function for different values of the parameters. It is obvious from this figure that the congestion function can generate the cost when the hub utilisation exceeds the capacity level. The congestion costs increase more and more rapidly as the hub utilisation increases. Moreover, a higher value for $b$ means faster cost growth.

\subsection{Notation}

Generally, each transit service is characterised by a set of technical (such as speed level, capacity, and departure frequency) and commercial (such as

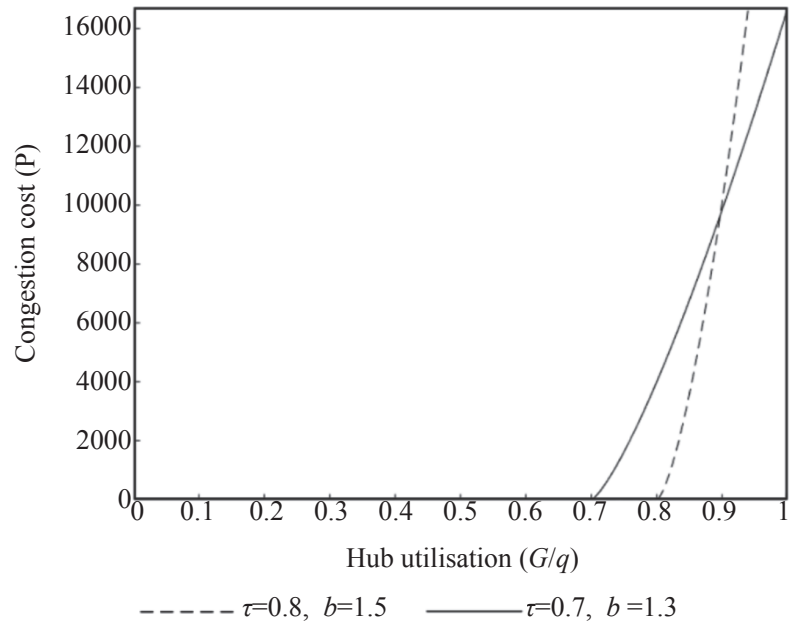

Figure 2 - Power-law function $(e=10, q=1000)$

service range and fares) characteristics. It is essential to design hierarchical passenger hubs to serve the system better because of the hierarchical nature of transit services. To illustrate our problem in details, the notations including the required data, decision variables, and intermediate variables are as follows.

\section{Required data:}

$I \quad$ - set of demand nodes, $I=\{1,2, \ldots,|I|\}$.

$J$ - set of candidate locations, $J=\{1,2, \ldots,|J|\}, J \subseteq I$.

$J_{e}$ - set of existing hubs, $J \subseteq J$.

$H$ - set of levels, $H=\{1,2, \ldots,|H|\}$.

$L-$ set of demand types, $L=\{S, S H\}$.

$w_{i h}^{l}-$ the level- $h \in H$ demand of type $l \in L$ originating at node $i \in I$.

$d_{i j}$ - the distance between demand node $i \in I$ and potential hub node $j \in J$.

$d_{h}^{l}-$ the travel distance of level- $h \in H$ demand of type $l \in L$.

$r_{h}$ - the maximum acceptable distance for level- $h \in H$ demand to access a hub.

$q_{k}$ - the maximum capacity of a level- $k \in H$ hub.

$f_{k}$ - the fixed cost of constructing a level- $k \in H$ hub.

$\beta_{j}-$ the regional cost differences at node $j \in J$. 
$m_{j}$ - the level of the existing hub $j \in J_{e}, m_{j} \in H$.

$a_{j}$ - the salvage value from selling the existing hub $j \in J_{e}$.

$c_{0}$ - the transportation cost of unit distance by arcs between a node and hub.

$c_{k}$ - the unit costs per distance under level- $k \in \mathrm{H}$ hub service.

$\delta_{i}$ - the value of time of passengers from node $i \in I$, unit: Chinese Yuan (CNY).

$v_{0}$ - the travel speed of arcs between a node and hub.

$v_{k}$ - the travel speed of level- $k \in H$ service.

Decision variables:

$o_{j}=\left\{\begin{array}{l}1 \text { if the existing hub } j \in J_{e} \text { is closed and sold } \\ 0 \text { if the existing hub } j \in J_{e} \text { remains open }\end{array}\right.$.

$x_{j k}=\left\{\begin{array}{l}1 \text { if a level }-k \in H \text { hub is located at } j \in J \\ 0 \text { otherwise }\end{array}\right.$.

$y_{i h j}^{l}$ - the proportion of the level- $h \in H$ demand of type $l \in L$ originating at node $i \in I$ that receives service from hub $j \in J$.

Intermediate variables:

$C_{i h j}^{l}$ - the transportation cost that the level- $h \in H$ demand of type $l \in L$ from node $i \in I$ spends via hub $j \in J, C_{i h j}^{l}=c_{0} d_{i j}+c_{k} d_{h}^{l}$.

$T_{i h j}^{l}$ - the total travel time that the level- $h \in H$ demand of type $l \in L$ from node $i \in I$ spends via hub $j \in J, T_{i h j}^{l}=d_{i j} / v_{0}+\left(d_{h}^{l}\right) v_{k}$.

\subsection{Model formulation}

To design an efficient megaregional hub system, we consider two strategic decisions: determining the locations and levels of hubs and allocating demand nodes to the located hubs. The hub locations include both existing and proposed new alternative locations. One of the options in the location decision is to sell the existing hub and benefit from its salvage value. We assume that a new hub at a different level can be built on the location of a sold hub when necessary.

When making allocation decisions, we assume that passengers only access their nearby hubs within a certain acceptable distance. With regard to the limited capacity of hubs, the congestion effects on each hub must also be taken into account. In other words, the goal in this paper is to determine which hubs to open and their optimal levels so that passengers are assigned to one or more opened hubs while minimising the total cost associated with hub location, congestion effects, and demand transportation costs.

The model formulation is as follows:

$$
\begin{aligned}
& \operatorname{minimize} \sum_{j \in J} \sum_{k \in H} x_{j k} \beta_{j} f_{k}-\sum_{j \in J_{e}}\left(1-o_{j}\right) \beta_{j} f_{m_{j}}-\sum_{j \in J_{e}} o_{j} a_{j} \\
& +\sum_{h \in H} \sum_{l \in L} \sum_{i \in I} \sum_{j \in J} y_{i h j}^{l} w_{i h}^{l}\left(C_{i h j}^{l}+\delta_{i} T_{i h j}^{l}\right)+\sum_{j \in J} P_{j} \\
& \text { subject to: } \\
& \sum_{k \in H} x_{j k} \leq 1 \quad \forall j \in J \\
& x_{j m_{j}}=1-o_{j} \quad \forall j \in J_{e} \\
& \sum_{j \in J} y_{i h j}^{l}=1 \quad \forall i \in I, h \in H, l \in L \\
& y_{i h j}^{S} \leq x_{j h} \quad \forall i \in I, h \in H, j \in J \\
& y_{i h j}^{S H} \leq \sum_{h \leq k \leq h+1} x_{j k} \quad \forall i \in I, h \in H, h \neq|H|, j \in J \\
& y_{j h j}^{S} \geq x_{j h} \quad \forall h \in H, j \in J \\
& \left(d_{i j}-r_{h}\right) y_{i h j}^{l} \leq 0 \quad \forall i \in I, h \in H, l \in L, j \in J \\
& G_{j}=\sum_{i \in I} \sum_{h \in H} \sum_{l \in L} y_{i h j}^{l} w_{i h}^{l} \quad \forall j \in J \\
& G_{j}<x_{j k} q_{k} \quad \forall j \in J \\
& P_{j}=\max \left\{0, e\left(G_{j}-\tau x_{j k} q_{k}\right)^{b}\right\} \quad \forall j \in J, k \in H \\
& o_{j} \in\{0,1\} \quad \forall j \in J_{e} \\
& x_{j k} \in\{0,1\} \quad \forall j \in J, k \in H \\
& y_{i h j}^{l} \geq 0 \quad \forall i \in I, h \in H, l \in L, j \in J
\end{aligned}
$$

The objective function 1 is composed of five terms. The first term is the total construction cost of all hubs, including new hubs and existing hubs. However, the construction cost of an existing hub should be subtracted from the first term if this existing hub remains open, so the second term needs to be deducted when $o_{j}$ is equal to zero. The third term of the objective function calculates the salvage value in the case that the existing hub should be closed and sold. The benefit from the salvage value is considered as a cost reduction because the objective function minimises the cost. The first three terms of the objective function 1 are actually the investment cost of the decision makers. We consider the transportation cost and time cost of passengers in the fourth term of the objective function. The last term represents the hub congestion cost.

Constraints 2 guarantee that different levels of hubs cannot be located at the same node. Constraints 3 assure that the decision variable $x_{j m_{j}}$ of an existing hub is linked to the variable $o_{j}$. If $o_{j}$ is equal to zero, the level of an existing hub remains unchanged. 
Conversely, if $o_{j}$ is equal to one, it is possible to open a new hub with a different level at the location of an existing hub.

Constraints 4 ensure that the sum of the fractions for the chosen hub is one. Constraints 5 and 6 indicate that passengers can receive service only from located hubs with the corresponding level. The type $S$ demand must receive the same level of hub service. However, the level- $h$ demand of type $S H$ can be provided by hubs at level $h$ or level $h+1$. Constraints 7 ensure that the type $S$ demand originating at node $j$ only receives service from hub $j$ when node $j$ is a hub at the same level. Constraints 8 are used to prevent any fraction of level- $h$ demand from node $i$ to choose hub $j$, when hub $j$ is out of the allowed area.

Constraints 9 represent the total demand served by hub $j \in J$. Constraints 10 require that the travel demand served by a hub does not exceed its maximum capacity. Constraints 11 represent the congestion cost of hub $j \in J$, where e, $\tau$ and $b$ are positive constants. Finally, constraints $12-14$ are domain constraints.

The above model formulation is a mixed-integer nonlinear programming (MINLP) problem due to the convex property of the congestion functions but the convexity of the congestion term is a desirable feature since we can linearise the formulation by a piecewise linear approximation [19]. This approximation allows us to transform the model to a mixed-integer linear programming (MILP) problem. It can be solved by commercial solvers in a straightforward way.

\section{COMPUTATIONAL STUDY}

In this section, a series of computational experiments were conducted to illustrate the performance of the proposed model on a real-world problem. The experiments were run on a personal computer with an Intel Core i7, 2.6 GHz CPU, 16 GB RAM, and Windows 10 Pro as the operating system. We formulate the model as an MINLP problem, which is transformed to an MILP problem using the toolbox YALMIP R20200116 [20] together with MATLAB R2019a. Gurobi v8.1.1 is utilised as the MILP solver. The optimal solution is obtained within a reasonable computational time (less than $2 \mathrm{~h}$ for each experiment).

\subsection{A real-world case study}

The study area is the WHM, which is located in the eastern Hubei Province in Central China, covering $58,000 \mathrm{~km}^{2}$. This area has been divided into 36 population centres, including 9 cities, with a total population of 32.1 million in 2018. A geographical map, including the population centres and existing transportation hubs, is shown in Figure 3.

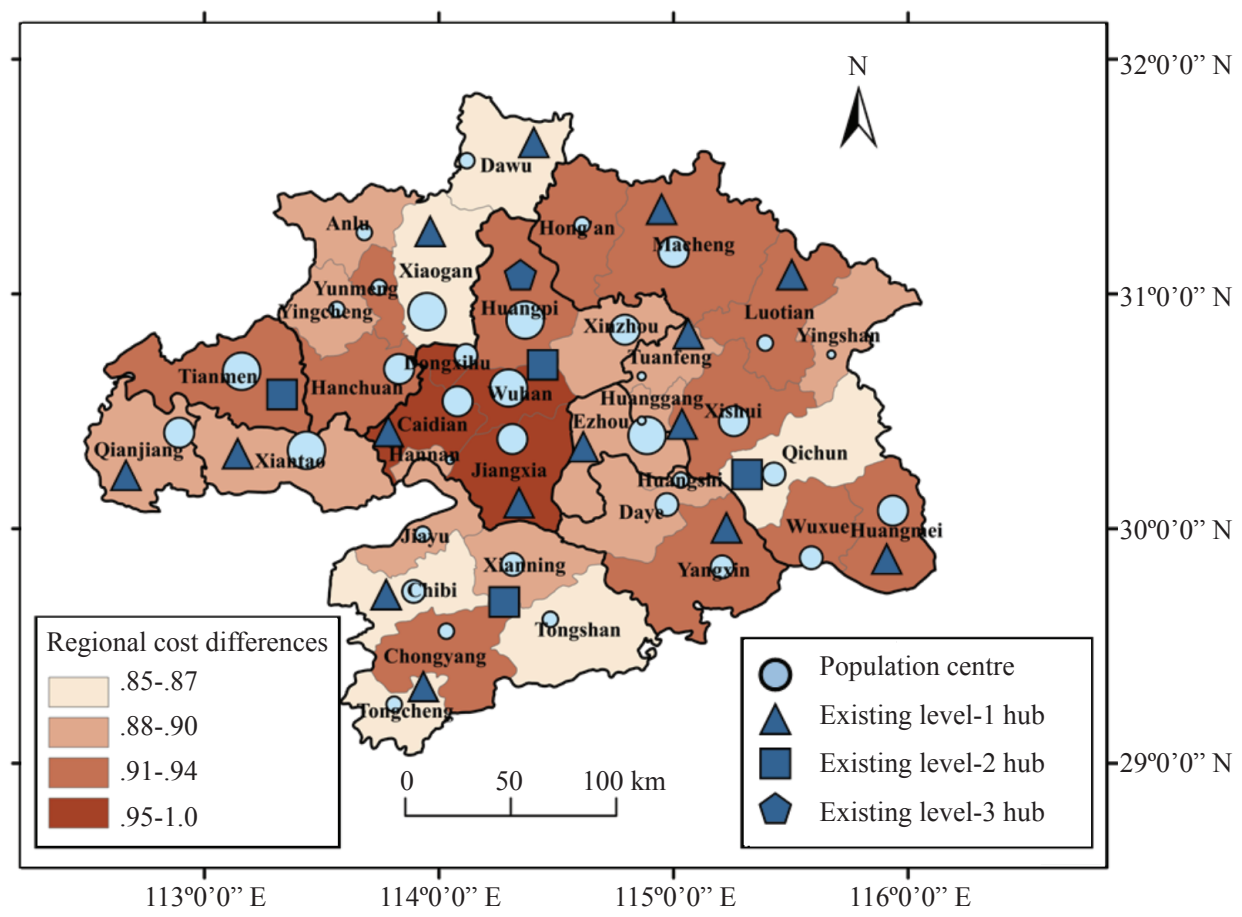

Figure 3 - Geographical map of the WHM and its existing transportation hubs 
In this paper, each population centre is considered as a demand node with different demand generation rates according to its population. We consider three levels of hubs for megaregional transportation networks, namely intercity bus hubs, railway hubs, and airline hubs. Each transportation mode has an optimal service range for market coverage. The fixed construction cost to establish a new hub is determined based on the regional cost differences (Figure 3) and its level. We use the peak-hour capacity as the maximum capacity for hubs. For the sake of clarity, only one kind of vehicle is considered for each transportation mode. The parameter values for hubs at each level are shown in Table 1.

Based on the distance intervals of the transportation services, the travel demand of each node can be divided into (i) level-1 demand (below $200 \mathrm{~km}$ ), (ii) level-2 demand (between 200 and $1500 \mathrm{~km}$ ), and (iii) level-3 demand (over $1500 \mathrm{~km}$ ). According to this classification, we can see that an intercity bus is the only choice for short-distance travel that is below $50 \mathrm{~km}$. For trips between 50 and $200 \mathrm{~km}$, both the level-1 and level-2 transportation modes can meet the demand within this range. Hence, the level-1 demand is separated into type $S$ demand (below $50 \mathrm{~km}$ ), and type $S H$ demand (50 $200 \mathrm{~km}$ ). Similarly, the travel distances of type $S$ and type $S H$ demand at level 2 are set as $200-800$ $\mathrm{km}$ and $800-1500 \mathrm{~km}$, respectively. When the travel distance is greater than $1500 \mathrm{~km}$, such as for international travel, an airplane is the only available transportation mode. Because the amount of travel

Table 1 - Parameter values for hubs at each level

\begin{tabular}{||l|c|c|c||}
\hline \multirow{2}{*}{\multicolumn{1}{c|}{ Parameters }} & \multicolumn{3}{|c||}{ Level } \\
\cline { 2 - 4 } & $k=1$ & $k=2$ & $k=3$ \\
\hline \hline Service range $[\mathrm{km}]$ & $(0,200)$ & $(5,1500)$ & $(800, \sim)$ \\
\hline$f_{k}\left[10^{6} \mathrm{CNY}\right.$ per day $]$ & 5 & 60 & 80 \\
\hline$q_{k}\left[10^{4}\right.$ persons per day $]$ & 5 & 20 & 10 \\
\hline$v_{k}[\mathrm{~km} / \mathrm{h}]$ & 70 & 200 & 900 \\
\hline$c_{k}[\mathrm{CNY} / \mathrm{km}]$ & 0.32 & 0.45 & 0.68 \\
\hline
\end{tabular}

demand varies by the range of travel distance, we set the proportions of demand for the five distance intervals mentioned above to $15 \%, 35 \%, 35 \%, 10 \%$, and $5 \%$, respectively.

Regarding the access part of a trip, we assume that the maximum acceptable distances for demand at each level to access a hub are $50 \mathrm{~km}, 100 \mathrm{~km}$, and $200 \mathrm{~km}$, respectively. Note that we address two basic access modes in the experiments according to the access distance. The corresponding parameters are set as $c_{0}=0.2, v_{0}=30\left(d_{i j} \leq 50\right)$ and $c_{0}=0.6, v_{0}=50$ $\left(d_{i j}>50\right)$. The distance between each pair of nodes is obtained from the road distance between the two population centres. The time value of passengers depends on the per-capita GDP at each centre.

We first focus on analysing the effect of congestion on the optimal location-allocation plan. It is worth mentioning that the capacity level $\tau$ can be considered an optimal utilisation rate of design capacity since above this level congestion starts to rise. The case where hubs at each level have different values of $\tau$ can reflect the characteristics of hub service. Thus the values of $\tau$ at each level are set to $85 \%, 80 \%$, and $75 \%$, respectively. Regarding the choice of $e$ in the power-law congestion function, we use $e=10$ in all experiments.

Table 2 presents the system cost changes under different congestion effects. The experiments were devised without congestion and then using $b=\{1.0,1.2,1.4,1.6\}$. The congestion effects are enhanced as the parameter $b$ increases. Note that TC, IC, TRC, and CC represent the total cost, investment cost, transportation cost, and congestion cost, respectively. As mentioned above, the investment cost is the first three terms of the objective function 1 . As shown in Table 2, the total cost increases as the parameter $b$ increases, since considering congestion effects not only induces congestion cost but also adjusts the optimal location-allocation plan to address congestion. More specifically, the cost associated with investment varies according to the location plan, and the transportation cost fluctuates with the demand allocation. Overall, the results

Table 2 - Impact of congestion on the system cost

\begin{tabular}{||c|c|c|c|c|c|c||}
\hline \multicolumn{2}{|c|}{$b$} & No congestion & 1.0 & 1.2 & 1.4 & 1.6 \\
\hline \hline \multirow{3}{*}{ Cost (CNY) } & TC & $623,220,941$ & $625,702,847$ & $640,014,319$ & $668,794,158$ & $692,046,688$ \\
\cline { 2 - 7 } & IC & $161,168,829$ & $161,168,829$ & $166,748,108$ & $195,423,088$ & $236,217,667$ \\
\cline { 2 - 7 } & TRC & $462,052,113$ & $462,061,901$ & $461,523,871$ & $464,011,417$ & $455,821,818$ \\
\cline { 2 - 7 } & CC & 0 & $2,472,117$ & $11,742,341$ & $9,359,653$ & 7,203 \\
\hline
\end{tabular}




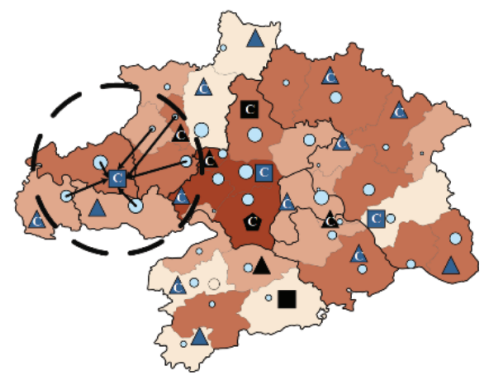

a) No congestion

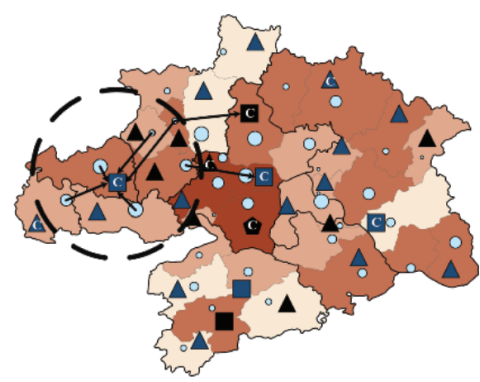

d) $b=1.4$

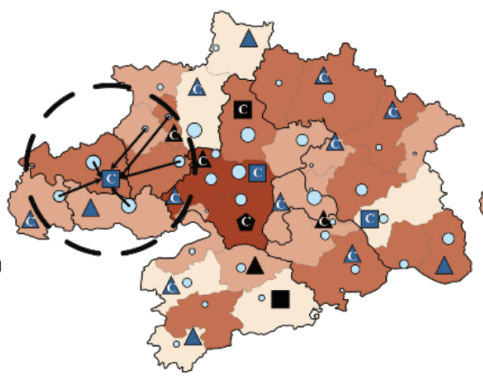

b) $b=1.0$

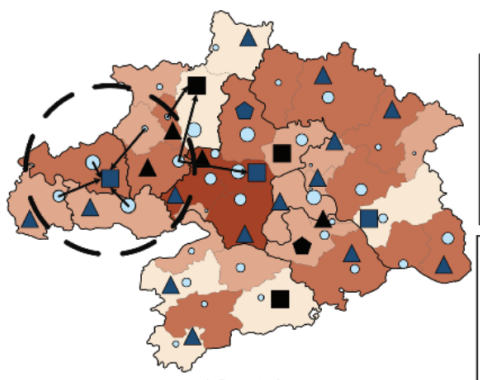

e) $b=1.6$

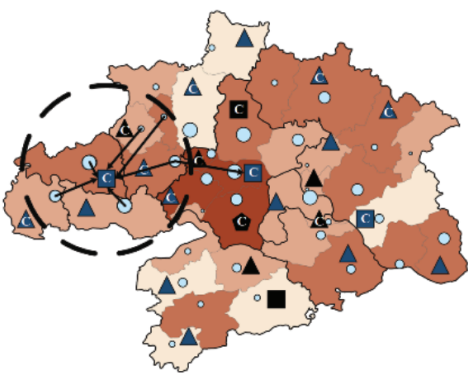

c) $b=1.2$

Figure 4 - Optimal solutions for the case study with different parameter values

indicate that the proposed system can be expected to perform well by considering the congestion effects in the model.

Figure 4 shows the optimal solutions for the case study. For brevity, we present only partial results of the allocation plan. In Figure 4, a congested hub, marked by the letter ' $\mathrm{C}$ ', is exceeding its capacity level by $1 \%$ or more. We can see that most of the hubs $(74 \%)$ are congested, when congestion effects are not considered or light congestion effects $(b=1.0)$ are assumed. However, the number of congested hubs decreases as the value of $b$ increases because as can be observed in Figure $4 d$ and $4 e$ : (i) the former instance establishes the largest number of hubs, which can yield a lower average demand served by each hub; (ii) only the latter instance has two level-3 hubs, which achieve a balanced distribution of level-3 demand. Demand allocation also plays a significant role in preventing the over-utilisation of hubs. For example, Figures $4 a-4 c$ show that six demand nodes receive service from the level-2 hub within the dashed circle. However, as shown in Figure $4 d$ and $4 e$, the number of demand nodes allocated to this hub decreases from 5 to 4 . This indicates that the proposed model can produce a new allocation plan to relieve hub congestion.

To further investigate this foregoing reduction in the hub congestion cost, we present the results of the hub congestion cost at each level, the number of hubs at each level, and the ratio of the demand served by hubs at each level in Table 3. As expected, the congestion effects can be alleviated by constructing new

Table 3 - Impact of congestion on the number of hubs and demand ratio

\begin{tabular}{||l|l|c|c|c|c|c||}
\hline \multicolumn{2}{|c|}{$b$} & No congestion & 1.0 & 1.2 & 1.4 & 1.6 \\
\hline \hline \multirow{4}{*}{$k=1$} & Congestion cost & 0 & 718,184 & $1,831,110$ & $1,279,723$ & 4,802 \\
\cline { 2 - 8 } & Number of hubs & 17 & 17 & 19 & 22 & 19 \\
\cline { 2 - 8 } & Demand ratio [\%] & 40.69 & 40.69 & 43.11 & 45.56 & 40.79 \\
\hline \multirow{3}{*}{$k=2$} & Congestion cost & 0 & $1,599,600$ & $9,012,664$ & $2,066,229$ & 2,401 \\
\cline { 2 - 8 } & Number of hubs & 5 & 5 & 5 & 6 & 6 \\
\cline { 2 - 8 } & Demand ratio [\%] & 54.20 & 54.20 & 51.89 & 49.44 & 54.21 \\
\hline \multirow{3}{*}{$k=3$} & Congestion cost & 0 & 154,333 & 898,566 & $6,013,700$ & 0 \\
\cline { 2 - 8 } & Number of hubs & 1 & 1 & 1 & 1 & 2 \\
\cline { 2 - 8 } & Demand ratio [\%] & 5.11 & 5.11 & 5.00 & 5.00 & 5.00 \\
\hline
\end{tabular}


hubs. For instance, for $k=1$ and when $b$ is increased from 1.2 to 1.4 , the hub congestion cost is reduced from $1,831,110$ to $1,279,723$. The same effect can also be seen in the cases of the level-2 (see rows 5 and 6) and level-3 hubs (see rows 6 and 7). Additionally, an interesting observation from columns 3, 6, and 9 is that when the value of $b$ increases, the order of construction is the lower-level hub first followed by the higher-level hub. This is because the construction cost of the former is lower than that of the latter. These instances present a good trade-off between congestion cost and construction cost in detail.

Another interesting observation from columns 4 and 7 is that the demand ratio varies with the number of hubs. More specifically, the demand served by level-1 hubs first increases and then decreases, while the demand served by level- 2 hubs changes in the reverse order. This is because adding another hub provides more demand allocation options. Consequently, after new hubs are constructed, the type $S H$ demand at level 1 would be allocated to these new hubs to avoid the congestion cost.

In general, decision makers focus not only on the demand allocation of the hubs at each level but also on the utilisation of every single hub. The utilisation of a hub $\frac{G_{j}}{x_{j k} q_{k}}$ is the ratio of the total demand served by the hub to its maximum capacity. Table 4 displays the maximum and minimum utilisation of the hubs at each level and the hub imbalance ratio, measuring the ratio of the maximum to the minimum hub utilisation. This table shows that a large hub imbalance ratio exists in two cases: one without considering congestion effects or where light congestion effects $(b=1.0)$ are assumed and the other where new hubs have just been added to the network (a level- 1 or 2 hub with $b=1.4$ and a level-3 hub with $b=1.6$ ). It is clear that building a new hub can increase the capacity of the network but with a lower hub utilisation, while when the congestion effect is ignored, a fully laden hub can be observed.

Furthermore, we observe that for the same number of hubs as shown in Table 3, the imbalance ratio of hubs (except the level-3 hub) decreases as $b$ increases, which implies that giving consideration to congestion leads to a balanced distribution of flow among hubs. Therefore, the hubs are utilised under their capacity levels when $b=1.6$. However, we can see that the imbalance ratio of level-1 hubs cannot drop to its minimum value of 1 . This is because the maximum acceptable distance for demand at each level to access a hub is introduced to limit the amount of covered demand. At this point, the minimum hub utilisation (see column 3) may fluctuate around a constant value but is not equal to the maximum hub utilisation.

\subsection{Improvement of the access service}

In principle, the accessibility of hubs has a significant impact on the utilisation of a transportation network. A transportation management agency can increase the attractiveness of public transportation by improving the access service such as increasing the access speed. Because the maximum acceptable distance for demand to access a hub is closely related to the access time, we vary the access speed $\left(v_{0}\right)$ and the maximum acceptable distances $\left(r_{h}\right)$ together to observe their effect on the resulting solutions of our model.

In this section, we consider four levels of access service. The speed of two basic access modes ranges from 30,50 to $45,65 \mathrm{~km} / \mathrm{h}$, respectively, in increments of 5 . The corresponding values of $r_{h}$ are $(50,100,200)$, $(60,120,240),(70,140,280)$ and $(80,160,320)$. All the experiments are conducted with $b=1.6$.

Table 4 - Impact of congestion on hub utilisation

\begin{tabular}{|c|c|c|c|c|c|c|}
\hline & $b$ & No congestion & 1.0 & 1.2 & 1.4 & 1.6 \\
\hline \multirow{3}{*}{$k=1$} & $\operatorname{Max}[\%]$ & 100.0 & 100.0 & 95.3 & 90.9 & 85.0 \\
\hline & $\operatorname{Min}[\%]$ & 36.5 & 36.5 & 36.5 & 33.4 & 36.5 \\
\hline & Hub imbalance ratio & 2.74 & 2.74 & 2.61 & 2.72 & 2.33 \\
\hline \multirow{3}{*}{$k=2$} & $\operatorname{Max}[\%]$ & 100.0 & 100.0 & 96.7 & 81.2 & 80.0 \\
\hline & $\operatorname{Min}[\%]$ & 79.3 & 79.3 & 79.3 & 34.2 & 79.3 \\
\hline & Hub imbalance ratio & 1.26 & 1.26 & 1.22 & 2.37 & 1.01 \\
\hline \multirow{3}{*}{$k=3$} & $\operatorname{Max}[\%]$ & 90.4 & 90.4 & 88.4 & 88.4 & 53.0 \\
\hline & $\operatorname{Min}[\%]$ & 90.4 & 90.4 & 88.4 & 88.4 & 35.5 \\
\hline & Hub imbalance ratio & 1.00 & 1.00 & 1.00 & 1.00 & 1.49 \\
\hline
\end{tabular}



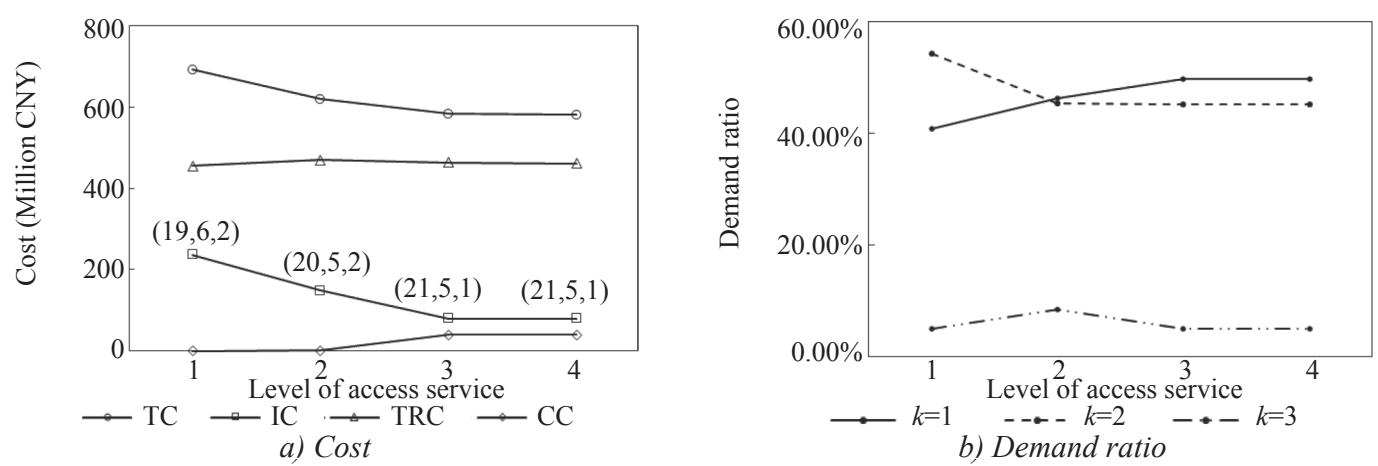

Figure 5 - Impact of access service on the system

Figure 5 illustrates the impact of access service on the network. The number of hubs at each level is marked on the IC line. Figure $5 a$ shows that the improvement of access service causes considerable decreases in the investment cost and total cost of the system. When the maximum acceptable distances $\left(r_{h}\right)$ increase, passengers can select hubs located much farther away, and this phenomenon tends to reduce the number of hubs with a higher construction cost. For example, the number of level-2 hubs decreases from 6 to 5 when the level of access service increases from 1 to 2 . Figure $5 b$ also shows that the type $\mathrm{SH}$ demand served by level-2 hubs is allocated to level-1 and level-3 hubs. We can see the same effect when the level of access service increases to 3 , reducing the number of level-3 hubs. But Figure $5 a$ shows that these changes also cause overloading of the hubs, resulting in congestion cost. Therefore, it can be concluded that improving the access service decreases the investment cost, but actually increases the congestion cost as well.

For completeness, we summarise the results of hub utilisation in Table 5. In general, we see that this strategy realises an increase in the minimum utilisa- tion of level-1 hubs but also leads to the over-utilisation of level-3 hubs. We can conclude that improvements to hub accessibility would increase the utilisation of passenger hubs.

\subsection{Planning of comprehensive transportation hubs}

In most location models, hubs of different levels cannot be located at the same node. However, there has been increased interest in planning comprehensive transportation hubs that integrate at least two transportation modes [21]. For example, the Hongqiao Integrated Transportation Hub in Shanghai combines multiple transportation modes, such as air, railways, and intercity buses. For this purpose, we consider the planning of comprehensive transportation hubs in the WHM. Constraints 2 are replaced by the following constraints in this case:

$\sum_{k \in H} x_{j k} \leq 3 \quad \forall j \in J$

We also implement an experiment with $b=1.6$. Figure 6 shows the location results of the new model. We find that there are five nodes with comprehensive

Table 5 - Impact of access service on hub utilisation

\begin{tabular}{||c|l|c|c|c|c||}
\hline \multicolumn{2}{|c|}{ Level of access service } & 1 & 2 & 3 & 4 \\
\hline \hline \multirow{3}{*}{$v_{0}[\mathrm{~km} / \mathrm{h}]$} & M1 & 30 & 35 & 40 & 45 \\
\cline { 2 - 6 } & M2 & 50 & 55 & 60 & 65 \\
\hline \multirow{2}{*}{$r_{h}[\mathrm{~km}]$} & $(50,100,200)$ & $(60,120,240)$ & $(70,140,280)$ & $(80,160,320)$ \\
\hline \multirow{5}{*}{$k=1$} & Max [\%] & 85.0 & 85.4 & 85.1 & 85.1 \\
\cline { 2 - 6 } & Min [\%] & 36.5 & 36.5 & 67.3 & 67.3 \\
\cline { 2 - 6 } & Hub imbalance ratio & 2.33 & 2.34 & 1.26 & 1.26 \\
\hline \multirow{5}{*}{$k=2$} & Max [\%] & 80.0 & 80.1 & 80.0 & 80.0 \\
\cline { 2 - 6 } & Min [\%] & 79.3 & 80.1 & 80.0 & 80.0 \\
\cline { 2 - 6 } & Hub imbalance ratio & 1.01 & 1.00 & 1.00 & 88.4 \\
\hline \multirow{3}{*}{$k=3$} & Max [\%] & 53.0 & 75.1 & 88.4 & 88.4 \\
\cline { 2 - 6 } & Min [\%] & 35.5 & 75.0 & 88.4 & 1.00 \\
\cline { 2 - 6 } & Hub imbalance ratio & 1.49 & 1.00 & 1.00 & \\
\hline
\end{tabular}




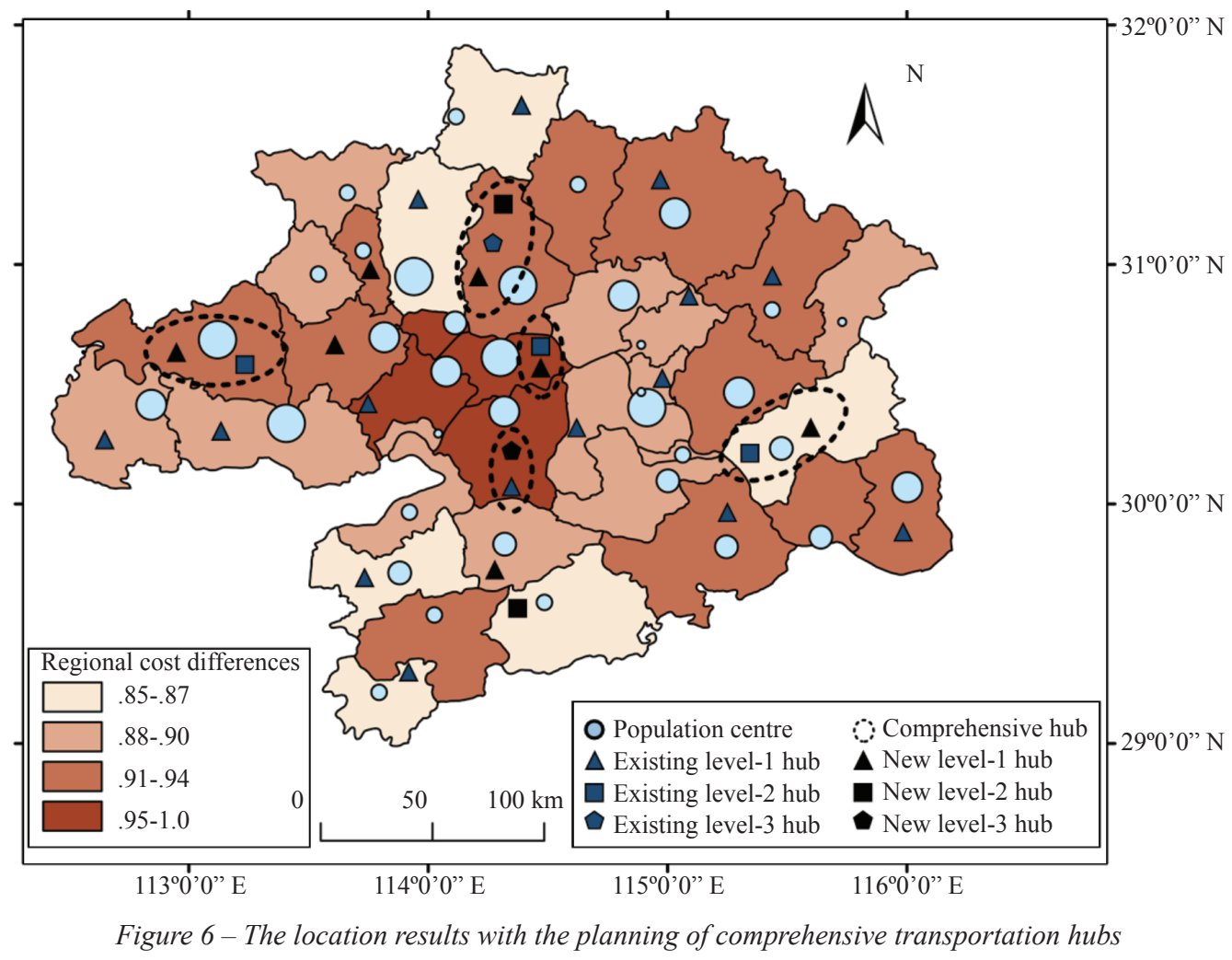

transportation hubs. Specifically, one air-railwaybus hub, one air-bus hub, and three railway-bus hubs are established in this solution. Compared to that in the location plan shown in Figure $4 e$, the number of level-2 hubs decreases from 6 to 5 , and all hubs are still utilised below their capacity levels. Moreover, the investment cost of the new model decreases by $16.96 \%$. This is because constraint 15 can improve land space utilisation, providing more candidate locations for hub facilities.

However, the passenger hubs would be located together in fewer locations with the planning of comprehensive transportation hubs, and this limitation increases the access distance of passengers. Additionally, the travel cost increases by $2.05 \%$. These results show that establishing comprehensive transportation hubs might be unfair to some passengers. However, overall, the total cost of the new model is $4.44 \%$ less than that of the basic model. Thus, the planning of comprehensive transportation hubs is a strategy to improve location solutions under congestion effects.

\section{CONCLUSIONS AND FUTURE RESEARCH}

This paper describes a megaregional passenger transportation system with hierarchical hub facilities. We study the hierarchical hub facility location problem with a new service availability structure and consider congestion effects on hubs. Specifically, a crossed service availability structure is introduced based on the relationship between intercity travel demand and hub facility service. Hub congestion is modelled through a convex function that increases exponentially when exceeding a given flow threshold. We develop a mixed-integer nonlinear programming model that combines decisions of closing existing hubs and selecting the locations of new hubs of different levels. Meanwhile, passengers are allocated to meet the hierarchical travel demands. The objective function is to minimise total cost, including investment cost, transportation cost, and congestion cost.

To evaluate the efficiency and effectiveness of the proposed model, we firstly solve five instances with different parameters in the congestion function. We consider three types of hubs and three levels of demand to model a megaregional passenger transportation system. The results show that the congestion effects can be addressed by reallocating the demand to balance the hub utilisation, and constructing new hubs to increase the network capacity. The trade-offs among transportation costs, investment costs, and congestion costs are well implemented. In the latter studies, we implement some instances to analyse the effects of the two strategies. The results show that 
the improvement of access service can increase hub utilisation, and the system cost decreases due to a significant decrease in the investment cost, and the planning of comprehensive transportation hubs can improve land space utilisation, which also decreases the investment cost. Overall, the computational results and the case analysis can be applied by decision makers to adopt appropriate strategies to optimise megaregional passenger transportation networks.

Future studies can be conducted to consider the congestion effect on the number of passengers. In fact, the congestion cost in this paper is undertaken by the hub operator and passengers. The travel demand at each node would decrease with this cost. Hence, the topic of the hub facility location problem under demand uncertainty requires further investigation.

\section{ACKNOWLEDGEMENTS}

This study has been substantially supported by the National Natural Science Foundation of China through several projects (Grant Nos. 72021002 and 71890973) and a project sponsored by the program of Shanghai academic research leader (No. 19XD1403800).

\section{间黄，博士生 ${ }^{1}$}

邮箱: tjyh@tongji.edu.cn

张小宁, 博士 ${ }^{1}$

(通讯作者)

邮箱: cexzhang@tongji.edu.cn

1 同济大学经济与管理学院, 中国上海市四平路 1239 号

\section{考虑拥挤效应的城市群客运交通枢纽选址问题 摘要}

由于最近的研究表明日益增长的城际出行需求影 响了城市群运输系统的效率, 因此在城市群范围内 制定有效的交通枢纽规划变得越来越重要。本文研 究了城市群客运交通网络中的分层设施选址问题。 研究的目的是确定不同层级枢纽设施的位置, 并以 最小的总成本（包括投资，运输和拥堵成本）将客 运需求分配到这些设施上。考虑到服务可用性结构 和枢纽的拥挤效应, 我们将此问题建模为混合整数 非线性规划模型。通过对武汉城市群的案例研究以 证明该模型的有效性。结果表明, 可以通过重新分 配需求以平衡枢纽的利用率或通过新建枢纽以增加 网络容量来解决拥挤问题。最后本文提出了最优的 枢纽位置和出行需求分配的方案, 以优化城市群内 的客运交通网络, 这对决策者具有重要意义。

\section{关键词}

\section{层级枢纽设施选址；城市群客运交}

通；优化；服务可得性；枢纽拥挤

\section{REFERENCES}

[1] Hagler Y. Defining U.S. Megaregions. America 2050; 2009. Available from: https://rpa.org/uploads/ pdfs/2050-Paper-Defining-US-Megaregions.pdf

[2] Yu J, et al. Cluster-based hierarchical model for urban transit hub location planning: Formulation, solution, and case study. Transportation Research Record. 2009;2112(1): 8-16. DOI: 10.3141/2112-02

[3] Contreras I, Fernández E. General network design: A unified view of combined location and network design problems. European Journal of Operational Research. 2012;219(3): 680-697. DOI: 10.1016/j.ejor.2011.11.009

[4] Rodrigue J-P, et al. The Geography of Transportation Systems. Hofstra University, Department of Global Studies \& Geography; 2020. Available from: https://transportationgeography.org

[5] Farahani RZ, et al. Hierarchical facility location problem: Models, classifications, techniques, and applications. Computers \& Industrial Engineering. 2014;68: 104-117. DOI: 10.1016/j.cie.2013.12.005

[6] Farahani RZ, et al. Hub location problems: A review of models, classification, solution techniques, and applications. Computers \& Industrial Engineering. 2013;64(4): 1096-1109. DOI: 10.1016/j.cie.2013.01.012

[7] Narula SC. Hierarchical location-allocation problems: A classification scheme. European Journal of Operational Research. 1984;15(1): 93-99. DOI: 10.1016/03772217(84)90052-3

[8] Zarrinpoor N, Fallahnezhad MS, Pishvaee MS. The design of a reliable and robust hierarchical health service network using an accelerated benders decomposition algorithm. European Journal of Operational Research. 2018;265(3): 1013-1032. DOI: 10.1016/j.ejor.2017.08.023

[9] Marianov V, Serra D. Hierarchical location-allocation models for congested systems. European Journal of Operational Research. 2001;135(1): 195-208. DOI: 10.1016/S0377-2217(00)00314-3

[10] Grove PG, O'Kelly ME. Hub networks and simulated schedule delay. Papers in Regional Science. 1986;59(1): 103-119. DOI: 10.1111/j.1435-5597.1986.tb00985.x

[11] Elhedhli S, Hu FX. Hub-and-spoke network design with congestion. Computers \& Operations Research. 2005;32(6): 1615-1632. DOI: 10.1016/j.cor.2003.11.016

[12] Gillen D, Levinson D. Full cost of air travel in the California corridor. Transportation Research Record. 1999;1662(1): 1-9. DOI: 10.3141/1662-01

[13] Elhedhli S, Wu H. A Lagrangean heuristic for hub-andspoke system design with capacity selection and congestion. INFORMS Journal on Computing. 2010;22(2): 282296. DOI: $10.1287 /$ ijoc. 1090.0335

[14] De Camargo RS, et al. Multiple allocation hub-and-spoke network design under hub congestion. Computers \& Operations Research. 2009;36(12): 3097-3106. DOI: 10.1016/ j.cor.2008.10.004

[15] De Camargo RS, de Miranda Jr G, Ferreira RPM. A hybrid outer-approximation/benders decomposition algorithm for the single allocation hub location problem 
under congestion. Operations Research Letters. 2011;39(5): 329-337. DOI: 10.1016/j.orl.2011.06.015

[16] De Camargo RS, Miranda G. Single allocation hub location problem under congestion: Network owner and user perspectives. Expert Systems with Applications. 2012;39(3): 3385-3391. DOI: 10.1016/ j.eswa.2011.09.026

[17] Alumur SA, et al. Modeling congestion and service time in hub location problems. Applied Mathematical Modelling. 2018;55: 13-32. DOI: 10.1016/j.apm.2017.10.033

[18] Özgün-Kibiroğlu Ç, Serarslan MN, Topcu Yİ. Particle swarm optimization for uncapacitated multiple allocation hub location problem under congestion. Expert Systems with Applications. 2019;119: 1-19. DOI: 10.1016/j.eswa.2018.10.019

[19] Alkaabneh F, Diabat A, Elhedhli S. A Lagrangian heuristic and GRASP for the hub-and-spoke network system with economies-of-scale and congestion. Transportation Research Part C: Emerging Technologies. 2019;102: 249-273. DOI: 10.1016/j.trc.2018.12.011

[20] Lofberg J. YALMIP: A Toolbox for Modeling and optimization in MATLAB. In: Proceedings of 2004 IEEE International Conference on Robotics and Automation, New Orleans, LA; 2004. p. 284-289.

[21] Huang HJ, et al. Transportation Issues in Developing China's Urban Agglomerations. Transport Policy. 2020;85: A1-A22. DOI: 10.1016/j.tranpol.2019.09.007 